\title{
TO A QUESTION ON THE EXISTENCE OF REGULAR MOSAICS ON A HYPERBOLIC PLANE OF POSITIVE CURVATURE
}

\author{
L. ROMAKINA \\ Received 03 October, 2016
}

\begin{abstract}
A hyperbolic plane $\hat{H}$ of positive curvature can be realized on the exterior domain with respect to an oval curve in a projective plane. The plane $\hat{H}$ is the Cayley-Klein model of a two-dimensional de Sitter space. It is proved that there is no regular mosaic on the plane $\hat{H}$.
\end{abstract}

2010 Mathematics Subject Classification: 51M10; 51M20; 52C20

Keywords: hyperbolic plane of positive curvature, generalized polygon, regular mosaic, hyperbolic regular mosaic

\section{INTRODUCTION}

\subsection{The hyperbolic plane $\hat{H}$ of positive curvature}

Let $H^{2}$ be an extended hyperbolic plane with absolute oval curve $\gamma$ [41, §5.1.1]. Every line on the plane $H^{2}$ belongs to one of three types depending on its position with respect to the absolute. Lines intersecting the absolute in two real (imaginary conjugate) points are called hyperbolic (elliptic). Any isotropic line on $\mathrm{H}^{2}$ tangent to the absolute is called parabolic.

In the Cayley-Klein model the interior domain with respect to the oval curve $\gamma$ is a complete Lobachevskii plane $\Lambda^{2}$ which is also called a Bolyai-Lobachevskian plane. This plane is the hyperbolic plane of negative curvature. On the set of all exterior points of the plane $H^{2}$ with respect to the absolute curve $\gamma$ one can construct different geometries. If we consider hyperbolic, elliptic, and parabolic lines as lines in our geometry, we obtain a hyperbolic plane $\hat{H}$ of positive curvature [31,32], [40, $\$ 4.1 .1]$. Geometry of the plane $\hat{H}$ of curvature $1 / \rho^{2}$ can be realized on the sphere of real radius $\rho$ in the pseudo-Euclidean space $\mathbb{R}_{1}^{3}$ with antipodal points identified. Therefore the plane $\hat{H}$ is the projective model of a two-dimensional de Sitter space $[10,14]$. The number $\rho$ is called the curvature radius of $\hat{H}$. The plane $\hat{H}$ is homeomorphic to the Möbius band without boundary. The group $G$ of projective automorphisms of the absolute oval curve $\gamma$ is the fundamental group of transformations for $\hat{H}, H^{2}$, and the Lobachevskii plane $\Lambda^{2}$. 


\subsection{Problem statement}

Let $\mathbb{K}$ be the Euclidean plane $\mathbb{R}^{2}$, the elliptic plane $\mathbb{E}^{2}$ or the Lobachevskii plane $\Lambda^{2}$.

A regular mosaic on the plane $\mathbb{K}$ consists of $q$ congruent regular $p$-gons which fit together round a common vertex without gaps and overlappings. The Schläfli symbol $\{p, q\}$ denotes the type of the regular mosaic. Each regular mosaic of the type $\{p, q\}$ determines the full group $[p, q]$ of its symmetries and generates the regular normal partition of the plane $\mathbb{K}[11],[12, \S 5.1]$.

Denote the measure of the interior angle at a vertex of a regular $p$-gon by $\varphi$. On the Euclidean plane we have $\varphi=\pi(1-2 / p)$. For the measure $\varphi$ on the plane $\mathbb{E}^{2}$ (or $\left.\Lambda^{2}\right)$ the inequality $\varphi>\pi(1-2 / p)$ (or $\varphi<\pi(1-2 / p)$ respectively) holds. Therefore the positive integers $p$ and $q$ from the Schläfli symbol of a regular mosaic in the plane K satisfy one of the following conditions:

(i) $(p-2)(q-2)<4$ if $\mathbb{K}$ is the elliptic plane $\mathbb{E}^{2}$;

(ii) $(p-2)(q-2)=4$ if $\mathbb{K}$ is the Euclidean plane $\mathbb{R}^{2}$;

(iii) $(p-2)(q-2)>4$ if $\mathbb{K}$ is the Lobachevskii plane $\Lambda^{2}$.

The conditions (i) - (iii) allow us to find all regular mosaics of the planes $\mathbb{E}^{2}, \mathbb{R}^{2}$, and $\Lambda^{2}$. According to the inequality (i) all "spherical mosaics" are devided into seven types: $\{2, q\},\{q, 2\},\{3,3\},\{3,4\},\{4,3\},\{3,5\},\{5,3\}$, where both of the Schläfli symbols $\{2, q\}$ and $\{q, 2\}$ determine infinite series of regular mosaics. Owing to the inequality (ii) there exist only three types of regular mosaics, $\{3,6\},\{4,4\},\{6,3\}$, on the Euclidean plane $\mathbb{R}^{2}$. The inequality (iii) does not impose restrictions for a types number of the "hyperbolic mosaics". This number is infinite.

Regular mosaics (in other terminology, regular honeycombs) first were mentioned in the works by Klein (1879) and Stringham (1880). Schlegel (1883) and Schläfli (1855) investigated these objects in classical spaces of constant curvature, that is, Euclidean, elliptic and Lobachevskii spaces (see review in [11,12]). The fundamental work of Poincare [28] has given base for the research of Fuchsian groups, that is, discrete transformations groups of the Lobachevskii plane $\Lambda^{2}$, and has generated thereby a particular interest to the "hyperbolic mosaics". This subject still attracts a lot of attention (see, for instance, $[3,4,16,26,27])$. There are also other geometrical objects closely connected with Fuchsian groups, in particular, Coxeter groups of the plane $\Lambda^{2}$ (see, for instance, $[8,18,20]$ ).

The development of geometry on the ideal domain of the Lobachevskii plane $\Lambda^{2}$ has set new goals. In academic discussion new issues have been raised. One of them can be formulated from general point of view: whether the geometry of the plane $\hat{H}$ can make a contribution to the theory of Fuchsian groups? The research of this issue has led to the partitions construction on the plane $\hat{H}$. At first the series of so-called simple partitions have been constructed [29,32]. A simple partition of the plane $\hat{H}$ or a figure on $\hat{H}$ is monohedral, that is, all its cells are congruent. A cell of a simple 
partition is simple 4-contour with the border consisting of four parabolic segments. In [29] the star of a regular odd-dimensional $n$-contour is studied. The star consists of $n$ congruent simple 4-contours adjoining on whole edges. The component 4-contours of the star locate out along an elliptic line. Three vertices of each component 4contour lie in a hypercycle. Such packing is a bit similar to packing of cells in a regular mosaic. But unlike a mosaic it is impossible to use the star as an independent cell of partition as the star is a topological equivalent of the Möbius band.

In Fig. 1 the star of a regular 9-contour is shown. The component 4-contours $Q_{1}$, $\ldots, Q_{9}$ of the star locate out along the elliptic line $l$. The hypercycle $\omega$ contains three vertices from each component 4-contour of the star.

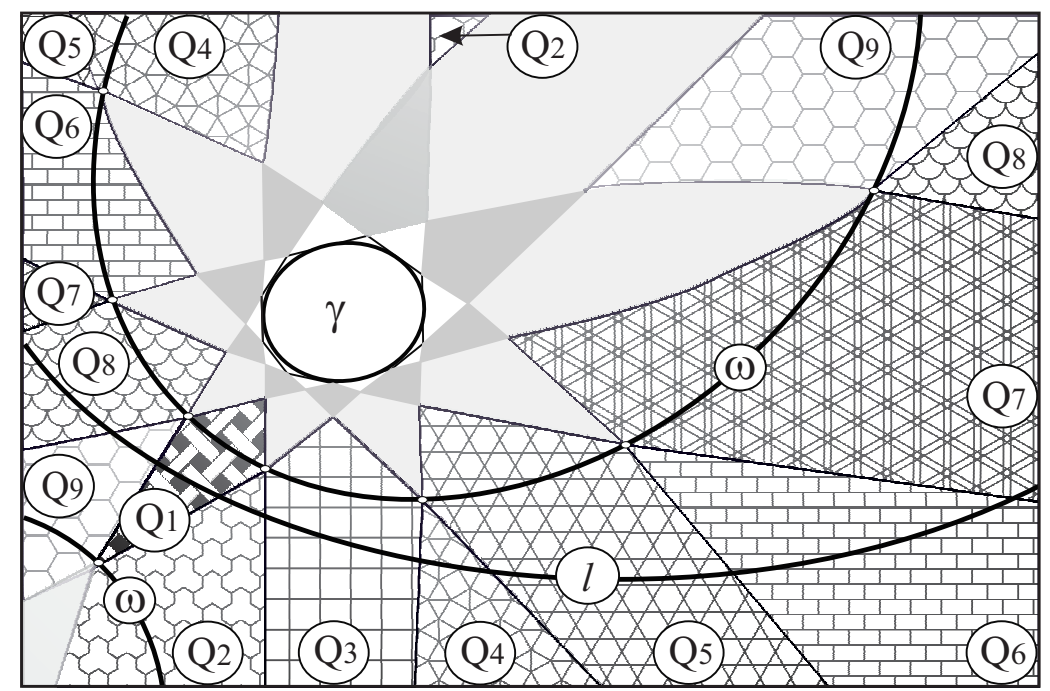

FIGURE 1. The absolute curve $\gamma$ and the star of a regular 9-contour. The component 4-contours $Q_{1}, \ldots, Q_{9}$ of the star.

We have also constructed an infinite tiling which may be considered as an remote analogue of a mosaic in the following sense: there exist both a simple partition of the tiling and a partition of the plane $\hat{H}$ by the tiling. Since a simple partition on the plane $\hat{H}$ is not normal [32, Theorem 5.1.1], the tiling significantly differs from a mosaic. In [34] the first normal fan partitions of the plane $\hat{H}$ are constructed. A fan partition is formed by special packing of congruent fans. Cells of a fan partition are also simple 4-contours, but not all cells are congruent. By Theorem 5.1.1 from [32] there is no regular mosaic consisting of simple 4-contours. Here we put more general question on the existence of regular mosaics. In Theorem 2 we prove that there is no regular mosaic on the plane $\hat{H}$. The simple proof of this fundamental fact is based on the theorem about the polygon area in the plane $\hat{H}$, see Theorem 3.1 in [38]. Since various stages of the proof of Theorem 3.1 have been published in three papers, we 
provide its short sketch in adjuvant Section 2. Besides, in Section 2 we give strict definitions of the notions used in this paper.

\section{PRELIMinaries}

\subsection{Angles of the plane $\hat{H}$}

There are three types of the pencils of lines on the plane $\hat{H}$. A pencil of lines on $\hat{H}$ is hyperbolic (elliptic) if its centre is an exterior (interior) point with respect to the absolute. The centre of a parabolic pencil lies on the absolute. Lines of a hyperbolic (elliptic) pencil are said to be intersecting (hyperparallel) on $\hat{H}$. Lines of a parabolic pencil are said to be parallel. Different variants of location of a pair of lines with respect to the absolute define fifteen types of angles of the plane $\hat{H}$ [30], [31, §4.3].

All types of angles of the plane $\hat{H}$ are presented in Table 1. We denote the types of pencils of lines by following symbols: $H$ - hyperbolic, $E-$ elliptic, $P-$ parabolic. For the types of lines in the plane $\hat{H}$ we use the following designations: $h$ - hyperbolic, $e$ - elliptic, $p-$ parabolic.

TABLE 1. The types and the measures of angles on the plane $\hat{H}$

\begin{tabular}{|c|c|c|c|c|}
\hline \multirow[t]{2}{*}{$\begin{array}{l}\text { Type of an angle } \\
\text { (short designation) }\end{array}$} & \multirow[t]{2}{*}{$\begin{array}{l}\text { Measure } v(\tilde{v}) \text { of } \\
\text { an angle }\end{array}$} & \multirow[t]{2}{*}{$\begin{array}{l}\text { Type of } \\
\text { a pencil }\end{array}$} & \multicolumn{2}{|c|}{$\begin{array}{l}\text { Types } \\
\text { of lines }\end{array}$} \\
\hline & & & $a$ & $b$ \\
\hline Valiana & - & \multirow[t]{2}{*}{$H$} & \multirow[t]{2}{*}{$p$} & \multirow[t]{2}{*}{$p$} \\
\hline Semicovaliana & - & & & \\
\hline Hyperbolic flag & - & \multirow[t]{2}{*}{$H$} & \multirow{3}{*}{$p$} & \multirow{3}{*}{$h$} \\
\hline Hyperbolic pseudoflag & - & & & \\
\hline Parabolic flag & - & $P$ & & \\
\hline Elliptic flag & - & \multirow[t]{2}{*}{$H$} & \multirow[t]{2}{*}{$p$} & \multirow[t]{2}{*}{$e$} \\
\hline Elliptic pseudoflag & - & & & \\
\hline Half-plane & $v \in[0 ; \pi]$ & $E$ & \multirow{5}{*}{$h$} & \multirow{5}{*}{$h$} \\
\hline Hyperbolic angle & $v \in \mathbb{R}_{+}$or $v_{a} \in \mathbb{R}_{-}$ & \multirow[t]{2}{*}{$H$} & & \\
\hline Hyperbolic pseudoangle & $\tilde{v}=i \pi+v, v \in \mathbb{R}_{+}$ & & & \\
\hline Strip & - & \multirow[t]{2}{*}{$P$} & & \\
\hline Pseudostrip & - & & & \\
\hline Quasiangle & $\begin{array}{c}\tilde{v}=\varepsilon v+i \pi / 2, \\
v \in \mathbb{R}_{+}, \varepsilon=1 ;-1 ; 0\end{array}$ & $H$ & $h$ & $e$ \\
\hline Elliptic angle & $v \in \mathbb{R}_{+}$ & \multirow[t]{2}{*}{$H$} & \multirow[t]{2}{*}{$e$} & \multirow[t]{2}{*}{$e$} \\
\hline Elliptic pseudoangle & $\tilde{v}=i \pi-v, v \in \mathbb{R}_{+}$ & & & \\
\hline
\end{tabular}

Angles of six types between non-parallel and non-parabolic lines in the plane $\hat{H}$ are measurable by means of the absolute. Measurable angles of five types possess 
vertices from the proper domain of the plane $\hat{H}$. Elliptic and hyperbolic angles, elliptic and hyperbolic pseudoangles, and quasiangles belong to such types. An elliptic angle has real positive measure while hyperbolic one has both positive measure and negative agreed measure. The measure of an elliptic pseudoangle is a number $i \pi-v$, and the measure of a hyperbolic pseudoangle is a number $i \pi+v$, where $v \in \mathbb{R}_{+}$.

Only non-parabolic lines of different types can be orthogonal on the plane $\hat{H}$. A line $a$ is orthogonal to a line $b$ if it contains a pole of the line $b$ with respect to the absolute. Two orthogonal lines form two adjacent right quasiangles. The measure of right quasiangle equals $i \frac{\pi}{2}$.

Assume that a hyperbolic line $a$ and an elliptic line $b$ form adjacent quasiangles with a common vertex $K$. Let $a^{\prime}$ be an orthogonal line to the line $a$ and let $K \in a^{\prime}$. The quasiangle between the lines $a$ and $b$ is called hyperbolic (or elliptic) if it contains (or, respectively, does not contain) the line $a^{\prime}$. The measure of a hyperbolic (elliptic) quasiangle is a number $i \frac{\pi}{2}+\varepsilon v$, where $v \in \mathbb{R}_{+}$and $\varepsilon=1(\varepsilon=-1)[31, \S 4.5]$.

\subsection{Polygons of the plane $\hat{H}$}

Let $\Phi$ be a figure of the plane $H^{2}$. If $\Phi \cap \hat{H}=\Phi(\Phi \cap \hat{H} \neq \Phi)$, then the figure $\Phi$ is called a finite (infinite) figure on $\hat{H}$.

In this paper we assume that the absolute curve $\gamma$ is added to the extended hyperbolic plane $H^{2}$. Such gluing the components $\hat{H}$ and $\Lambda^{2}$ of the plane $H^{2}$ by means of the curve $\gamma$ allow us to simplify the introduction of basic objects. Let $\bar{H}^{2}=\gamma \cup H^{2}$.

On the plane $\bar{H}^{2}$ there are two types of simple closed curves. A simple closed curve is called one-sided if its removal from $\bar{H}^{2}$ does not break connectivity of the plane $\bar{H}^{2}$. A simple closed curve is called two-sided if it divides the plane $\bar{H}^{2}$ into two connected components.

Let $F$ be a simple closed two-sided broken line on the plane $\bar{H}^{2}$ and let $F \cap \hat{H} \neq$ $\varnothing$. Then $F$ divides the plane $\bar{H}^{2}$ into two connected components. One of these components is topological equivalent to an open disk. Denote it by $o$. The second component is topological equivalent to the Möbius band without boundary. Denote it by $\mu$. The figure $F_{o}=F \cup o\left(F_{\mu}=F \cup \mu\right)$ is called a generalized polygon (generalized Möbius polygon) of the plane $\hat{H}$. The generalized polygon $F_{o}$ is also called a generalized polyhedral or a $n$-hedral depending on the number $n$ of its vertices.

Vertices and segments of the broken line $F$ are called respectively vertices and edges of the polygon with boundary $F$. A polygon vertex is called proper or ideal if it belongs to the plane $\hat{H}$ or to the plane $\Lambda^{2}$ respectively. A polygon vertex is called absolute if it lies on the absolute curve $\gamma$. A polygon vertex is called true in case the polygon edges containing this vertex do not lie on the same line.

Let $F$ be a generalized polygon or a generalized Möbius polygon of the plane $\hat{H}$ and let all vertices of the polygon $F$ be proper and true. Assume that lines $b$ and $d$ 
contain edges of the polygon $F$ with a common vertex $A$. The lines $b, d$ and a polar line $a$ of the point $A$ with respect to the absolute $\gamma$ divide the plane $\bar{H}^{2}$ into four generalized trihedrals. We call them the basic quarters of the vertex $A$ and denote by $\eta_{j}, j=1,2,3,4$.

Assume that an angle $\eta$ of the plane $\bar{H}^{2}$ between the lines $b$ and $d$ contains the basic quarter $\eta_{j}$ of the vertex $A$. We define a type of the basic quarter $\eta_{j}$ as the type of the angle $\eta$. If the angle $\eta$ is measurable, we call its measure a measure of the basic quarter $\eta_{j}$. Let the figures of four sets

$$
\left(\eta_{1}^{1}, \ldots, \eta_{1}^{m}\right),\left(\eta_{2}^{1}, \ldots, \eta_{2}^{n}\right),\left(\eta_{3}^{1}, \ldots, \eta_{3}^{r}\right),\left(\eta_{4}^{1}, \ldots, \eta_{4}^{s}\right),
$$

where $m, n, r$, and $s$ are positive integers, be connected components of the figures $F \cap \eta_{1}, F \cap \eta_{2}, F \cap \eta_{3}$, and $F \cap \eta_{4}$ respectively. The set of figures (2.1) contains one or three figures possessing the point $A$ and a nonempty interior. Denote the quantity (sum) of these figures by $Q_{A}\left(F_{A}\right)$.

Let us consider all possibilities.

(1) Assume that $Q_{A}=1$. Then one of the basic quarters of the vertex $A$ contains the figure $F_{A}$. We call this quarter the interior angle of the polygon $F$ at the vertex $A$.

(2) Assume that $Q_{A}=3$. Then only one of the basic quarters of the vertex $A$ does not contain components of the figure $F_{A}$. Denote it by $\eta_{4}$. We call the sum of the quarters $\eta_{1}, \eta_{2}, \eta_{3}$ the interior angle of the polygon $F$ at the vertex $A$. If the basic quarters $\eta_{1}, \eta_{2}, \eta_{3}$ are measurable, we define the measure of the interior angle of the polygon $F$ at the vertex $A$ as the sum of the measures of these quarters.

In Fig. 2, a (b) the interior angle $\eta_{1}\left(\eta_{1} \cup \eta_{2} \cup \eta_{3}\right)$ of the polygon $F$ at the vertex $A$ is shown.

Under the condition $Q_{A}=1$ (or $Q_{A}=3$ ) the interior angle of the polygon $F$ at the vertex $A$ is convex (or, respectively, non-convex).

Remark 1. The sum of the measures of adjacent angles with a vertex at a proper point of the plane $\hat{H}$ is equal to $i \pi$. Therefore the sum of the measures of all basic quarters of a polygon vertex is equal to $2 i \pi$. This means that the measure of a full angle round proper point in the plane $\hat{H}$ equals $2 i \pi$.

In classical geometries of the planes of constant curvature $\mathbb{E}^{2}, \Lambda^{2}$, and $\mathbb{R}^{2}$ the notion "full angle round a point" is closely connected with the notion "rotation round a point". In the geometry of the plane $\hat{H}$ these notions significantly differ. Each line on the plane $\mathbb{E}^{2}, \Lambda^{2}$, or $\mathbb{R}^{2}$ under the rotation round an arbitrary proper point can cover the full plane and return to its initial location. Each non-parabolic line on the plane $\hat{H}$ under the rotation round an arbitrary proper point infinitely moves away from its initial location. Staying of the same type, the line under such rotation go to the parabolic line containing the centre of rotation. In this regard, the notion "full 


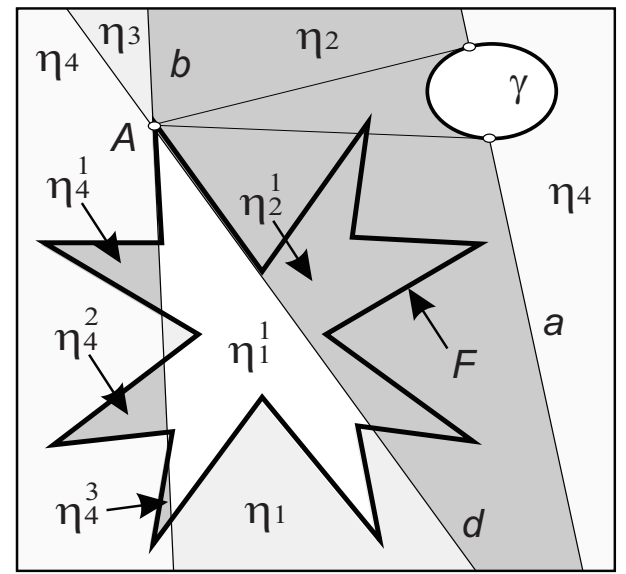

a

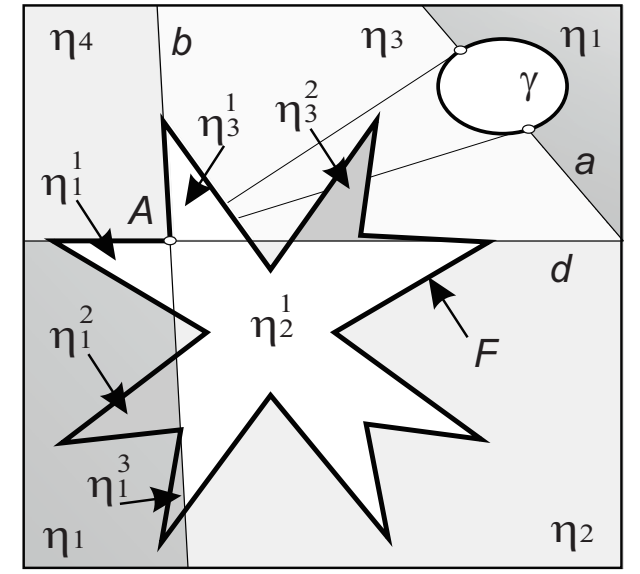

b

FIGURE 2. The absolute curve $\gamma$ and the interior angle $\eta_{1}\left(\eta_{1} \cup \eta_{2} \cup\right.$ $\eta_{3}$ ) of the polygon $F$ at the vertex $A(\mathrm{a})((\mathrm{b}))$.

angle round a point" in the geometry of the plane $\hat{H}$ possess conditional character. We apply this notion in the sense of the packing of figures round a point, that is, ignoring the rotation.

\subsection{Theorem on the area of a generalized polygon of the plane $\hat{H}$}

The foundation of the volumes theory, in particular, of the areas theory, in the hyperbolic space was given in classical works of the founders of non-Euclidean geometry, Lobachevsky and Bolyai (see, for instance, $[6,25]$ ). Later this theory was developed in works of many authors (see, for instance, [5, 7, 9, 19, 21, 42, 43]). Profound survey on this topic is presented in papers $[2,21,44]$.

It is known that on the plane $\Lambda^{2}$ of curvature $\left(-1 / \rho^{2}\right)$ the area of a triangle can be expressed through the measures $\hat{A}, \hat{B}$, and $\hat{C}$ of interior angles at vertices by the formula

$$
S=\rho^{2}(\pi-\hat{A}-\hat{B}-\hat{C}) .
$$

This formula for the first time was obtained by Lambert as a corollary of the hypothesis of the acute angle [24]. It is natural that considering projective model of the hyperbolic plane, some authors wondered about the truth of formula (2.2) on the extended hyperbolic plane $H^{2}$ for the generalized triangles with vertices both on interior, and on exterior domain of the plane with respect to the absolute (see, for instance, [5, 7, 43]). Unfortunately, the mistakes made by authors resulted in incorrect conclusions. Analyzing the known works, we emphasize two main problems. 
First, we note incorrect definitions of angles between lines in the plane $H^{2}$ (and $\hat{H})$. This problem is multilayered. Let us dwell on two important points in brief. Despite careful elaboration of this topic by leaders of geometry (see, for instance, $[1,17]$ ), some authors do not notice conceptual distinctions between the notions "angle" and "measure of an angle". Besides, some authors overlook the following fact. The plane $\hat{H}$ is homeomorphic to the Möbius band without boundary. Therefore adjacent angles between lines on $\hat{H}$ can be topologically distinct (see [31, \$4.3]). In this regard, the introduction of the basic notions in the plane $\hat{H}$ geometry demands extreme accuracy.

The second main problem consists in incorrect adaptation of Laguerre formulae [23] to the calculation of the measures of angles with vertices in the exterior domain with respect to the absolute of the plane $H^{2}$. Choosing a multiplier in the Laguerre formulae, authors recede from the general logic stated by Klein in [22]. We discuss this problem in [37].

Trying to improve the situation, we come back to basis of non-Euclidean geometry in its projective understanding. Basing on classical works [1,15, 17,22], we carefully define all basic notions of the plane $\hat{H}$ geometry (see [30-32]). Only after this we go to the calculation of the figures areas ${ }^{1}$.

The foundation of the areas theory of the plane $\hat{H}$ is set in works $[33,36,38,39]$. The notion of the area is introduced such that the finite figures of the plane $\hat{H}$ possess the real positive areas. Moreover, for each figure $F$ of the plane $H^{2}$ the following equality holds

$$
S_{\Lambda^{2}}(F)=i S_{\hat{H}}(F),
$$

where $S_{\Lambda^{2}}(F)$ or $S_{\hat{H}}(F)$ is the area of the figure $F$ calculated in geometry of the plane $\Lambda^{2}$ or $\hat{H}$ respectively (see $\left.[33,36]\right)$.

In this paper due to the theorem on the area of a generalized polygon without parabolic edges (see [38, Theorem 3.1]) we obtain the simple proof of the fundamental fact of the plane $\hat{H}$ geometry. Let us formulate this theorem.

Theorem 1. Let $F$ be a generalized polygon without parabolic edges on the plane $\hat{H}$ of curvature radius $\rho, \rho \in \mathbb{R}_{+}$. Denote the measures of interior angles of the polygon $F$ at vertices $A_{1}, A_{2}, \ldots, A_{n}$ by $\hat{A}_{1}, \hat{A}_{2}, \ldots, \hat{A}_{n}$ respectively. Then the area $S_{\hat{H}}(F)$ of the polygon $F$ can be expressed by the formula

$$
S_{\hat{H}}(F)=\rho^{2}\left(\sum_{j=1}^{n} \tau_{j} \hat{A}_{j}-i \pi(n-2)\right),
$$

\footnotetext{
${ }^{1}$ To create the theory of volumes on the ideal domain $\hat{H}^{3}$ of a hyperbolic space $H^{3}$ it is necessary to pass a similar way. We begin this way in works $[35,37]$. Notice that Gauss emphasized complexity of the volumes calculation in the hyperbolic space by the term "die Dschungel" (jungle). The volumes calculation in the space $\hat{H}^{3}$ is a much more complicated problem, because this space contains lines and planes of different topological types.
} 
where $\tau_{j}= \begin{cases}1 & \text { if } A_{j} \text { is a proper or absolute vertex of the polygon } F, \\ i & \text { if } A_{j} \text { is an ideal vertex of the polygon } F .\end{cases}$

Note that known formula (2.2) is a special case of formula (2.4). Indeed, by formula (2.4) for an arbitrary triangle $A B C$ in the plane $\Lambda^{2}$ with the measures $\hat{A}_{1}=\hat{A}$, $\hat{A}_{2}=\hat{B}$, and $\hat{A}_{3}=\hat{C}$ of interior angles at vertices $A, B$, and $C$ respectively, we have $\tau_{1}=\tau_{2}=\tau_{3}=i$ and

$$
S_{\hat{H}}(A B C)=\rho^{2}(i \hat{A}+i \hat{B}+i \hat{C}-i \pi) .
$$

From here via equality (2.3) we find the area of the triangle $A B C$ in the plane $\Lambda^{2}$ geometry:

$$
S_{\Lambda^{2}}(A B C)=i S_{\hat{H}}(A B C)=\rho^{2}(\pi-\hat{A}-\hat{B}-\hat{C}) .
$$

Thus for an arbitrary triangle in the plane $\Lambda^{2}$ formula (2.2) holds.

We prove Theorem 1 in three stages. At first, in paper [33], we choose an orthogonal hypercyclic coordinate system on the plane $\hat{H}$ and prove the following formula for the calculation of the area of a rectangular trihedral with an elliptic (hyperbolic) cathetus of length $a(b)$

$$
S=\rho^{2} \ln \frac{\sinh \frac{b}{\rho}+\cosh \frac{b}{\rho} \sin \frac{a}{\rho}}{\sin \frac{a}{\rho}+\cos \frac{a}{\rho} \sinh \frac{b}{\rho}} .
$$

After that, in [36], via formula (2.5) we prove the following formula for a finite trihedral of the plane $\hat{H}$ with the measures $\hat{A}_{1}, \hat{A}_{2}$, and $\hat{A}_{3}$ of interior angles

$$
S=\rho^{2}\left(\hat{A}_{1}+\hat{A}_{2}+\hat{A}_{3}-i \pi\right) \text {. }
$$

In article [38], we generalize formula (2.6). For this end we show that on the plane $\hat{H}$ there are 48 types of generalized trihedrals with non-parabolic edges. Hereafter for the generalized trihedral $A_{1} A_{2} A_{3}$ of each type we prove the formula

$$
S=\rho^{2}\left(\tau_{1} \hat{A_{1}}+\tau_{2} \hat{A_{2}}+\tau_{3} \hat{A_{3}}-i \pi\right),
$$

where $\tau_{j}=1\left(\tau_{j}=i\right)$ in case the point $A_{j}$ is the proper or absolute (ideal) vertex of the generalized trihedral $A_{1} A_{2} A_{3}$.

Finally, for a generalized polygon without parabolic edges after its triangulation and applying formula (2.7) to every cell of partition we obtain formula (2.4).

\section{MAIN THEOREM}

In this section we prove a theorem which reveals significant differences between the plane $\hat{H}$ geometry and the classical geometries of planes with constant curvature.

Theorem 2. There is no regular mosaic on the plane $\hat{H}$. 
Proof. I. At the first stage we prove the following assertion.

$\Delta:$ If there is a regular mosaic of the plane $\hat{H}$, then interior angles of its cells are right quasiangles.

Assume that there is a regular mosaic $\mathfrak{M}$ of the type $\{p, q\}$ on the plane $\hat{H}$. Let a proper point $A$ of the plane $\hat{H}$ be a common vertex of $q$ congruent regular $p$-hedrals $F_{1}, \ldots, F_{q}$ from the mosaic $\mathfrak{M}$. Denote the interior angle of the $p$-hedral $F_{j}$ at the vertex $A$ by $\alpha_{j}$, where $j=1, \ldots, q$. Let $k_{1}$ and $k_{2}$ be the parabolic lines passing through the point $A$. The lines $k_{1}, k_{2}$ contain four rays $k_{11}, k_{12}, k_{21}, k_{22}$ with a common origin $A$. The type of each angle $\alpha_{j}$ is unambiguously determined by its location with respect to these rays. Since the angles $\alpha_{1}, \ldots, \alpha_{q}$ are congruent, they are equally located with respect to the rays from the set $k_{11}, k_{12}, k_{21}, k_{22}$. In the sense of the task each angle $\alpha_{j}$ is convex and is not straight angle. Hence each angle $\alpha_{j}$ contains no more than two rays from the set $k_{11}, k_{12}, k_{21}, k_{22}$. Moreover, if the angle $\alpha_{j}$ contains two rays from this set, then the sides of the angle $\alpha_{j}$ contain two these rays. It is clear that the ray $k_{11}$ lies on the side or on the interior domain of some angle from the set $\alpha_{1}, \ldots, \alpha_{q}$. Denote this angle by $\alpha_{1}$. Then the side or, respectively, the interior domain of each of the angles $\alpha_{2}, \ldots, \alpha_{q}$ contains a ray from the set $k_{12}, k_{21}, k_{22}$. Consequently, the quantity of angles in the set $\alpha_{1}, \ldots, \alpha_{q}$ is equal to the quantity of rays in the set $k_{11}, k_{12}, k_{21}, k_{22}$. Therefore $q=4$ and a priori only two following cases are possible.

(1) Each of the rays $k_{11}, k_{12}, k_{21}, k_{22}$ lies on a common side of two adjacent angles from the set $\alpha_{1}, \alpha_{2}, \alpha_{3}, \alpha_{4}$ (Fig. 3, a).

(2) Each of the rays $k_{11}, k_{12}, k_{21}, k_{22}$ divides one of the angles $\alpha_{1}, \alpha_{2}, \alpha_{3}, \alpha_{4}$ (Fig. 3, b).

In the first case the angles $\alpha_{1}, \alpha_{2}, \alpha_{3}, \alpha_{4}$ are not congruent. Indeed, by the condition (1) the sides of the angles $\alpha_{1}, \alpha_{2}, \alpha_{3}, \alpha_{4}$ lie on the parabolic lines $k_{1}=k_{11} \cup k_{12}$ and $k_{2}=k_{21} \cup k_{22}$. Hence two angles from the set $\alpha_{1}, \alpha_{2}, \alpha_{3}, \alpha_{4}$ belong to the valiana of the point $A$ (see the angles $\alpha_{1}$ and $\alpha_{3}$ in Fig. 3, a). Two other angles belong to the covaliana of this point. Thus under the condition (1) the mosaic $\mathfrak{M}$ is not regular. This contradicts our assumption.

The second case does not lead to contradictions. Let us continue our reasonings, accepting the condition (2).

The sides of each angle $\alpha_{j}$ divide the pair of the parabolic lines $k_{1}, k_{2}$. Hence they are non-parabolic lines of different types. This means that each angle $\alpha_{j}$ is a quasiangle. Since the quasiangles $\alpha_{1}, \alpha_{2}, \alpha_{3}, \alpha_{4}$ are congruent, they are right quasiangles. The assertion $\Delta$ is proved.

II. Now we check the existence of a polygon which forms a regular mosaic on the plane $\hat{H}$. Considering the assertion $\Delta$, we choose the incomplete list of properties of such polygon, and we prove that this list contains incompatible requirements.

Assume that there is a polygon $F$ satisfying the following conditions.

(1) All interior angles of the polygon $F$ are right quasiangles. 


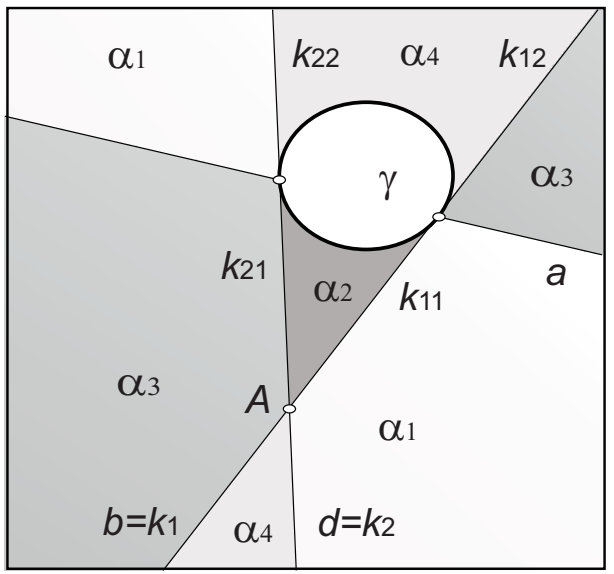

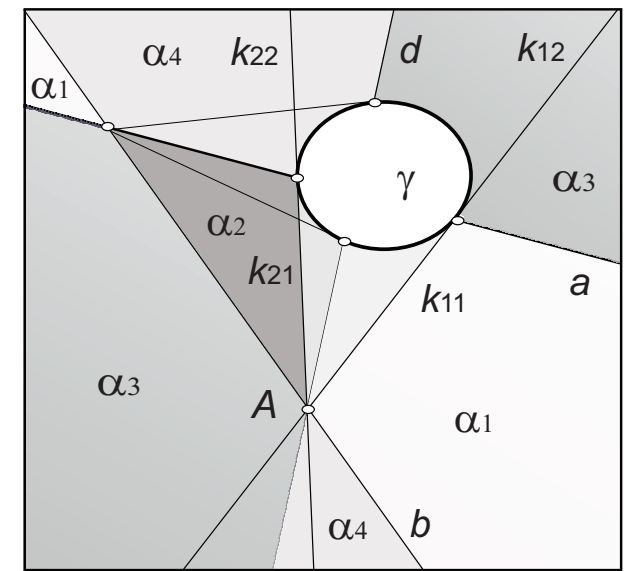

FigurE 3. The angles $\alpha_{1}, \alpha_{2}, \alpha_{3}, \alpha_{4}$ at the vertex $A$ of the regular mosaic $\mathfrak{M}$. The rays $k_{11}, k_{12}, k_{21}, k_{22}$ lie on the sides of the angles $\alpha_{1}, \alpha_{2}, \alpha_{3}, \alpha_{4}$ (a). The rays $k_{11}, k_{12}, k_{21}, k_{22}$ divide the angles $\alpha_{1}$, $\alpha_{3}, \alpha_{2}, \alpha_{4}$, respectively (b).

(2) The polygon $F$ is topological equivalent to a disk.

(3) The polygon $F$ is finite figure of the plane $\hat{H}$.

Denote the vertices of the polygon $F$ by $A_{1}, \ldots, A_{p}$. Under the condition (1) the measures $\hat{A}_{1}, \ldots, \hat{A}_{p}$ of the interior angles of the polygon $F$ at the vertices respectively $A_{1}, \ldots, A_{p}$ equal $i \pi / 2$.

Due to the requirement (2) the polygon $F$ satisfies the Theorem 1 conditions. By the condition (3) in the formulation of Theorem 1 for each vertex of the polygon $F$ we have $\tau_{j}=1$. Moreover, the area $S_{\hat{H}}(F)$ of the polygon $F$ is a real positive number. Formula (2.4) yields

$$
S_{\hat{H}}(F)=\rho^{2}\left(i \frac{\pi}{2} p-i \pi(p-2)\right)=\frac{i \pi \rho^{2}}{2}(4-p) .
$$

The expression (3.1) implies $S_{\hat{H}}(F) \in \mathbb{C}$ and $\operatorname{Re}\left(S_{\hat{H}}(F)\right)=0$. These facts contradict the notion of the area of a finite figure in the plane $\hat{H}$. Hence the conditions (1) - (3) are incompatible. So there is no polygon forming the regular mosaic on the plane $\hat{H}$. This yields the assertion of the theorem. The theorem is proved.

\section{COMPARATIVE ANALYSIS OF METHODS AND RESUlTS}

In Theorem 2 we proved that there is no regular mosaic on the plane $\hat{H}$. In the scientific discussion of this result the following question was raised: how the assertion about regular mosaics depends on the choice of the definition of angles on the 
plane $\hat{H}$. In Subsection 2.2 we have discussed the main problems of the definition of objects in hyperbolic geometry in this regard. Taking into account the prevalence of these problems, we show here, with specific examples, how they impede the solution of the questions about polygons and, in general, are slowing down the development of the plane $\hat{H}$ geometry.

While sharing the views expressed by Klein, we accept the following statement: Geometry is the totality of figures properties wich are invariant in transformations of some group. According to this statement we define geometrical figures with an accuracy to their congruence, understanding the congruence of geometrical figures as the possibility of their coincidence under some transformation of the space containing these figures. The congruence of geometrical figures differs from equality of the measures of these figures if they are measurable. Indeed, let us consider two segments of the length $a$ in the plane $\hat{H}$, where $a \in(0, \pi \rho)$. Assume that one of them is elliptic, and the second is hyperbolic. Having equal lengths, these segments are not congruent because any transformation of the group $G$ retains the line type. Now let us consider an elliptic angle and half-plane of the plane $\hat{H}$. Such geometrical figures can have equal measures from interval $(0, \pi)$ (see Table 1 and $[31, \S 4.5]$ ), but these figures are not congruent. The examples given show us that the information about the measure of a geometrical figure can't unambiguously characterize this figure. Therefore such information can't serve as the definition of a geometrical object.

Let us address, for example, Definition 2.1 of generalized angles on the hyperbolic plane from paper [13]. To reduce reasonings, we shall consider only objects of the plane $\hat{H}$. In our terms, in the case 1 (b) of Definition 2.1 two hyperbolic lines with the common point on exterior domain with respect to the absolute are presented. Such lines determine three objects on the plane $\hat{H}$. These objects are as follows: two congruent hyperbolic angles and hyperbolic pseudoangle (see angles $\alpha_{1}, \alpha_{2}$, and, respectively, $\alpha_{3}$ in Fig. 4, a). A hyperbolic angle and a hyperbolic pseudoangle are topologically distinct. In article [13] these objects are not distinguishable and geometrically are not defined. It is worth noticing that the phrase "their angle is the length" from Definition 2.1 is incorrect because an angle is a geometrical figure, and the length is a number.

In the case 2 of Definition 2.1 two elliptic lines are presented. Such lines determine elliptic angle and elliptic pseudoangle on the plane $\hat{H}$ (see angles $\alpha_{1}$ and $\alpha_{2}$ in Fig. 4 , b). These topologically different objects are not distinguishable and geometrically are not defined in paper [13] too. This means that at the using of Definition 2.1 the questions about polygons angles in the plane $\hat{H}$ will remain unresolved.

Formally Formula (6) from [13] yields the correct result only for the hyperbolic cosine of the measures of hyperbolic or elliptic angles and hyperbolic or elliptic pseudoangles. But Formula (6) does not give the actual values of the measures of these objects. Moreover, Remark 2.2 conclusively confuses the reader. The hyperbolic measures of adjacent or, in terms of paper [13], complementary angles except 


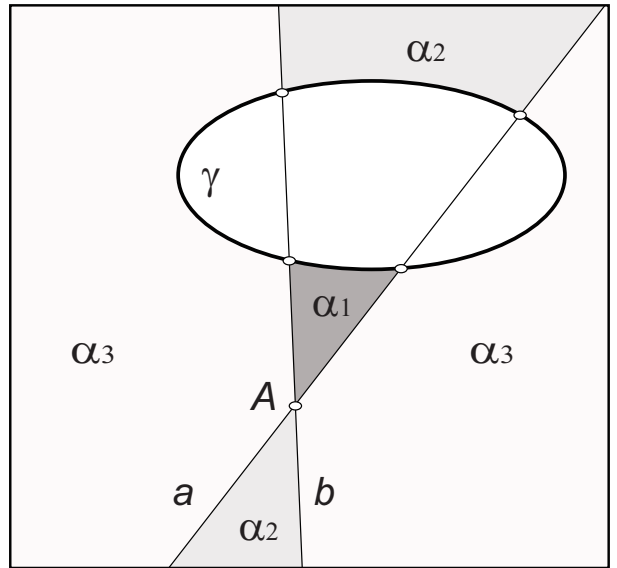

a

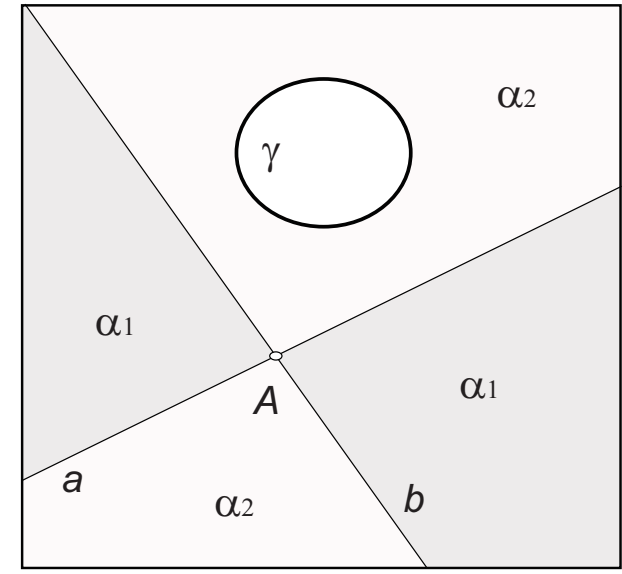

b

FIGURE 4. The hyperbolic angles $\alpha_{1}, \alpha_{2}$ and the hyperbolic pseudoangle $\alpha_{3}$ with the hyperbolic sides $a, b$ and the vertex $A$ (a). The elliptic angle $\alpha_{1}$ and the elliptic pseudoangle $\alpha_{2}$ with the elliptic sides $a, b$ and the vertex $A$ (b).

quasiangles are differ in their nature. The measure of a hyperbolic or elliptic angle is real, the measure of its adjacent pseudoangle is complex (see Table 1). The sum of the measures of adjacent angles is equal to $i \pi$ (see [31, \$4.5]). Therefore the using of real instead of complex measures is inadmissible. We notice also that the both signs \pm in Formula (6) is required because of the homogeneity of the coordinates used in this definition.

Thus Definition 2.1 from [13] is incomplete. On its basis it is impossible to investigate the polygons of the plane $\hat{H}$, in particular, it is impossible to prove Theorem 1 and to solve the question on the existence of regular mosaics.

The following full scheme of the introduction of geometrical objects was formed in classic geometrical works. We believe that this scheme will be useful to researchers in the field of non-Euclidean geometries.

(1) The geometrical definition of the class of congruent objects.

Definitions can be, for example, genetic, constructive, "through a sort and specific differences", by means of the classification.

(2) The indications and analytical conditions of the belonging of an arbitrary object to the entered class.

(3) The proof of the existence of an object from the entered class.

(4) The solution of the question about the possibility of the measurement for the entered objects.

(5) The geometrical definition of the measure of the entered objects.

(6) The determination of the admissible values of the entered measure. 
(7) The analytical expression of the entered measure in the choosen coordinate system.

\section{ACKNOWLEDGEMENT}

The author expresses her gratitude to Dr. Imre Juhász and the reviewer for their attention to this work and the interesting scientific discussion. Thanks to profound questions of the reviewer, this paper contains the analysis of the problems in the definition of geometrical figures.

\section{REFERENCES}

[1] M. Berger, Geometrie. Paris: Original French edition published by CEDIC and Fernand Nathan, 1977.

[2] N. V. Abrosimov, "On volumes of polyhedra in spaces of constant curvature." Bull. Kem. Un., vol. 3, no. 1, pp. 7-13, 2011.

[3] S. K. Baek, P. Minnhagen, and B. J. Kim, "Percolation on hyperbolic lattices." PRE, vol. 79, no. 1, p. 011124, 2009, doi: 10.1103/PhysRevE.79.011124.

[4] H. Belbachir, L. Németh, and L. Szalay, "Hyperbolic Pascal triangles." Applied Mathematics and Computation, vol. 273, pp. 453-464, 2016, doi: 10.1016/j.amc.2015.10.001.

[5] J. Böhm and H. C. Im Hof, "Fächeninhalt verallgemeinerter hyperbolische Dreiecke." Geom. Dedicata, vol. 42, pp. 223-233, 1992.

[6] J. Bolyai, Appendix. The Theory of Space Janos Bolyai (F. Kárteszi ed.). Budapest: Akadémiai Kiadó, 1987.

[7] Y. Cho, "Trigonometry in extended hyperbolic space and extended de Sitter space." Bull. Korean Math. Soc., vol. 46, no. 6, pp. 1099-1133, 2009, doi: 10.4134/BKMS.2009.46.6.1099.

[8] H. S. M. Coxeter, "Discrete Groups Generated by Reflections." Proc. Int. Congress Math., vol. 35, no. 3, pp. 588-621, 1934, doi: 10.2307/1968753.

[9] H. S. M. Coxeter, "The functions of Schläfli and Lobachefsky." Quart. J. Math. Oxford, vol. 6, no. 1, pp. 13-29, 1935.

[10] H. S. M. Coxeter, “A Geometrical Background for De Sitter's World.” Am. Math. Mon., vol. 50, no. 4, pp. 217-228, 1943.

[11] H. S. M. Coxeter, "Regular honeycombs in hyperbolic space." Proc. Int. Congress Math., vol. III, pp. 155-169, 1954.

[12] H. S. M. Coxeter and W. O. J. Moser, Generators and relations for discrete groups. New York: Springer-Verlag Berlin Heidelberg, 1972. doi: 10.1007/978-3-662-21946-1.

[13] G. Csima and J. Szirmai, "Isoptic curves of generalized conic sections in the hyperbolic plane." Manuscript, 2016. arXiv: 1504.06450.

[14] W. De Sitter, "On the Relativity of Inertia. Remarks Concerning Einstein's Latest Hypothesis." KNAW, Proceedings, vol. 19, no. 2, pp. 1217-1225, 1917.

[15] N. V. Efimov, Higher Geometry. Moscow: MAIK. Nauka/Interperiodika. FIZMATLIT, 2004.

[16] H. Gu and R. M. Ziff, "Crossing on hyperbolic lattices." PRE, vol. 85, no. III, p. 051141, 2012, doi: 10.1103/PhysRevE.85.051141.

[17] D. Hilbert, The foundations of geometry. Chicago: The Open Court Publishing Company, 1902.

[18] H.-C. I. Hof, “A class of hyperbolic Coxeter gpoups.” Expo. Math., vol. 3, pp. 179-186, 1985.

[19] W.-Y. Hsiang, "On infinitesimal symmetrization and volume formula for spherical or hyperbolic tetrahedrons.” Quart. J. Math. Oxford (2), vol. 39, pp. 463-468, 1988.

[20] S. B. Katok, Fuchsian groups. Chicago Lectures in Mathematics. Chicago: University of Chicago Press, 1992. 
[21] R. Kellerhals, "On the volume of hyperbolic polyhedra." Math. Ann., vol. 285, pp. 541-569, 1989.

[22] F. Klein, Vorlesungen Über Nicht-Euclidische Geometrie. Berlin: Verlag von Julius Springer, 1928.

[23] E. Laguerre, "Sur la theorie des foyers." Nouv. Ann. de Mathem., vol. 12, pp. 57-66, 1853.

[24] J. H. Lambert, Die Theorie der Parallellinien. $\quad$ Leipziger: Leipziger Magazin f ur Reine und Angewandte Mathematik, 1786.

[25] N. I. Lobachevsky, About the foundations of Geometry. Complete works. Vol. 1. Kazan: Kazan bulletin, 1829-1830.

[26] L. Németh, "Combinatorial examination of mosaics with asymptotic pyramids and their reciprocals in 3-dimensional hyperbolic space.” Stud. Sci. Math. Hung., vol. 43, no. 2, pp. 247-264, 2006 , doi: 10.1556/SScMath.43.2006.2.4.

[27] L. Németh, "Trees on hyperbolic lattices." Miskolc Math. Notes, vol. 16, no. 1, pp. 353-360, 2015.

[28] H. Poincaré, "Théorie des groups Fuchsiens.” Acta Math., vol. 5, pp. 1-62, 1882.

[29] L. N. Romakina, "Simple partitions of a hyperbolic plane of positive curvature." Mat. Sb., vol. 203, no. 9, pp. 83-16, 2012, doi: 10.1070/SM2012v203n09ABEH004266.

[30] L. N. Romakina, "Analogs of a formula of Lobachevsky for angle of parallelism on a hyperbolic plane of positive curvature." Sib. Elektron. Mat. Izv., vol. 10, pp. 393-407, 2013, doi: 10.17377/semi.2013.10.028.

[31] L. N. Romakina, Geometry of a hyperbolic plane of positive curvature. P. 1: Trigonometry. Saratov: Publishing House of the Saratov University, 2013.

[32] L. N. Romakina, Geometry of a hyperbolic plane of positive curvature. P. 2: Transformations and simple partitions. Saratov: Publishing House of the Saratov University, 2013.

[33] L. N. Romakina, "The theorem of the area of a rectangular trihedral of a hyperbolic plane of positive curvature." Dal'nevost. Mat. Zh., vol. 13, no. 1, pp. 127-147, 2013.

[34] L. N. Romakina, "Fan triangulations of a hyperbolic plane of positive curvature." Siberian Adv. Math., vol. 24, no. 3, pp. 204-221, 2014, doi: 10.3103/S1055134414030079.

[35] L. N. Romakina, "Classification of tetrahedrons with not hyperbolic sides in a hyperbolic space of positive curvature." Chebyshevskii Sb., vol. 16, no. 2, pp. 208-221, 2015.

[36] L. N. Romakina, "On the area of a trihedral on a hyperbolic plane of positive curvature." Sib. Adv. Math., vol. 25, no. 2, pp. 138-153, 2015, doi: 10,3103/S1055134415020042.

[37] L. N. Romakina, "Dihedrons of a Hyperbolic Three-Space of Positive Curvature." IEJG, vol. 9, no. 2, pp. 50-58, 2016.

[38] L. N. Romakina, "The area of a generalized polygon without parabolic edges of a hyperbolic plane of positive curvature." AJMCR (IKP), vol. 10, no. 4, pp. 293-310, 2016.

[39] L. N. Romakina, "To the theory of the areas of a hyperbolic plane of positive curvature." Publ. l'Inst. Math., vol. 99, no. 113, pp. 139-154, 2016, doi: 10.2298/PIM1613139R.

[40] B. A. Rosenfel'd, Noneuclidean spaces. Moscow: Nauka, 1969.

[41] B. A. Rosenfel'd and M. P. Zamakhovskii, Geometry of Lie groups. Symmetric, parabolic and periodic spaces. Moscow: Moscow Center for Countinuous Mathematical Education, 2003.

[42] L. Schläfli, Theorie der vielfachen Kontinuität. Gesammelte mathematishe Abhanlungen. Basel: Birkhäuser, 1950.

[43] J. M. Schlenker, "Metriques sur les polyedres hyperboliques convexes." J. Differential Geom., vol. 48, no. 2, pp. 323-405, 1998, doi: 10.4134/BKMS.2009.46.6.1099.

[44] E. B. Vinberg, "Volumes of non-Euclidean polyhedra." Uspekhi Mat. Nauk, vol. 48, no. 2(290), pp. 17-46, 1993. 
Author's address

\section{Romakina}

Saratov State University, Department of Geometry, 83 Astrahkanskaya St., 410012 Saratov, Russia

E-mail address: romakinaln@mail.ru 